\title{
PAPEL DA GASTROPLASTIA NO TRATAMENTO DA APNÉLA OBSTRUTIVA DURANTE O SONO
}

\author{
RUBENS REIMÃO * \\ HELIO LEMMI ** \\ GEORGE COWAN ** \\ EMMEL B. GOLDEN *;:
}

A redução de peso é tratamento complementar estabelecido para a sindrome de apnéia obstrutiva durante o sono (ASO)12,24. Por outro lado, muitas vezes esses pacientes têm obesidade severa e má aceitação da restrição dietética, a qual, junto com a necessidade de redução rápida de peso para alívio da disfunção respiratória, tornam tal medida inadequada. A gastroplastia 22,23 surgiu recentemente neste contexto como nova terapia auxiliar. Apresentamos aqui a evolução de pacientes com ASO e obesidade submetidos a gastroplastia. São focalizados os aspectos polissonográficos e a indicação terapêtica.

\section{CASUISTICA E METODOLOGIA}

Avaliamos 4 casos de ASO, todos do sexo masculino e obesos, cujos pesos eram $176 \%$ a $228 \%$ do peso ideal. As idades variaram de 31 a 54 anos. A investigação no centro de distúrbios do sono do Baptist Memorial Hospital constituiu-se de anamnese, exame clínico, neurológico e psiquiátrico, questionários para descrição dos hábitos de sono e um a três registros polissonográficos de noite inteira no pré-operatório imediato (Tabela 1), A montagem de tal exame incluia eletrencefalograma (EEG), eletrooculograma (EOG), eletrocardiograma, eletromiografia de submentonianos, pares termólétricos nasais e bucais para detecção de fluxo aéreo, pneumograma tưráxico e abdominal para análise de esforço respiratório. EEG foi realizado nas derivaçōes C3-A2 e C4-A1. EOG foi obtiđo tendo como referência Fp do EEG. A saturação arterial de oxigênio (Sa02) foi monitorizada de forma continua com detector transcutâneo acoplado ao polígrafo. Os ruidos respiratórios foram observados durante toda a noite e gravaram-je o comportamento e a respiração em videotape. As salas foram mantidas com isolamento acústico e temperatura em torno de $230 \mathrm{C}$.

Utílizamos critérios padronizados de Rechtschaffen e col. (17) para análise dos registros. Consideramos indice de apnéias (IA) normal (1a,11) quando ocorriam 5 ou menos apnéias por hora de sono. IA e o número de episódios apneicos por hora de sono (11). Nossos casos foram escolhidos para gastroplastia por terem obesidade acen-

Trabalho do Department of Neurodiagnostics, Baptist Memorial Hospital (BMH), Memphis, Tennessee, E.U.A.; * Neurologista; ** Professor de Neurologia, University of Tennessee; *** Cirurgião BMH, **** Pneumologista BMH, Bolsa CAPES 3502/82-3. 


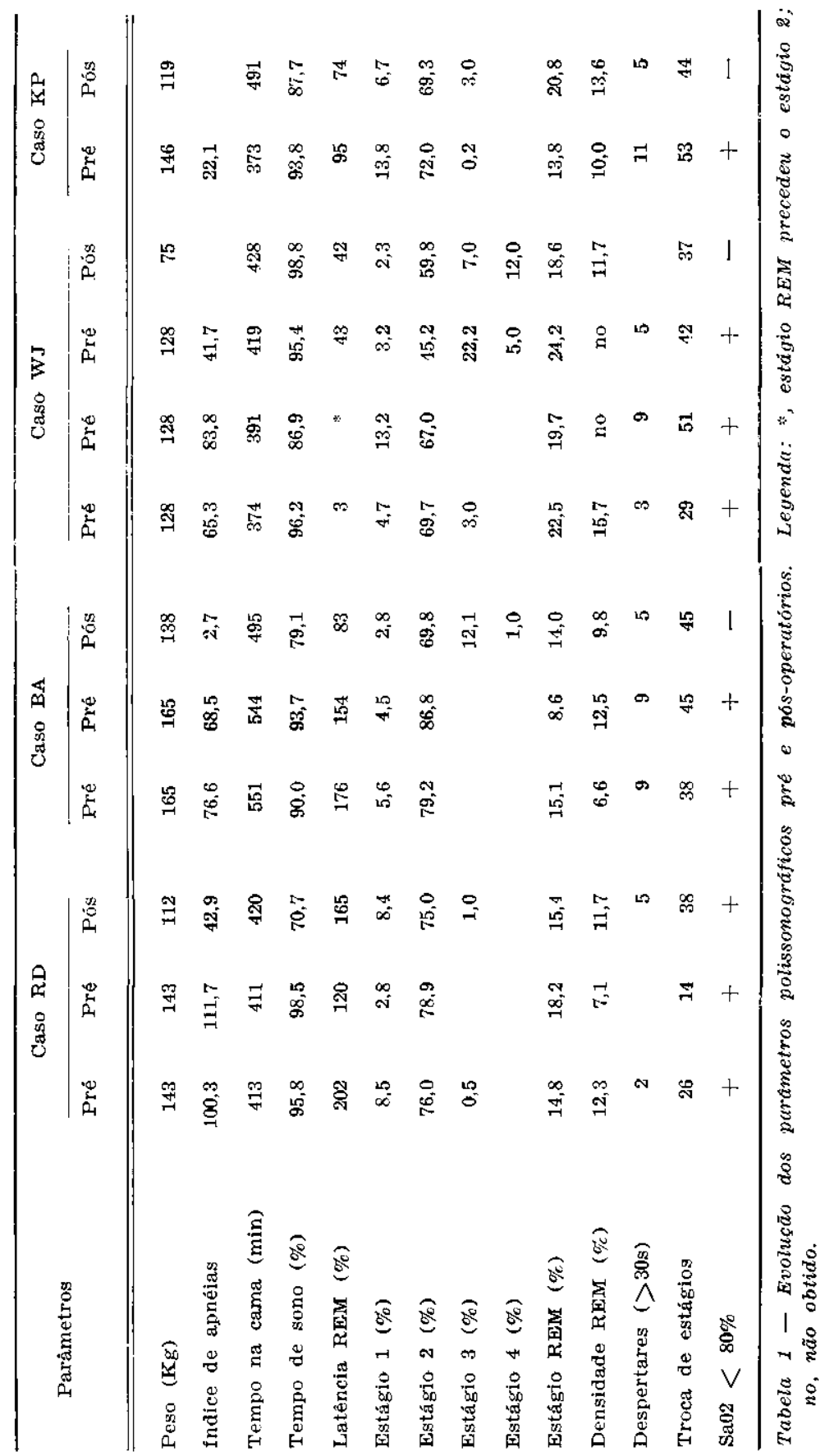


tuada, referirem incapacidade de seguimento de dietas, apresentarem ASO severa acompanhada de comprometimento cardio-respiratório e de redução acentuada da Sa02. Gastroplastia foi o único tratamento em um paciente (Caso WJ) e acompanhou-se de traqueostomia nos demais. A traqueostomia foi feita como medida temporária para alívio imediato do comprometimento cardio-respiratório pelas apnéias. Todos mantiveram traqueostomia fechada durante o dia e aberta ao dormir. Reavaliaçāo clínica e polissonográfica foi realizada 3 a 4 meses após a cirurgia. Tal registro, feito com traqueostomia fechada, seguiu os mesmos moldes do pré-operatório.

\section{RESULTADOS}

A queixa de sonolência excessiva diurna, roncar e pausas respiratórias clurante o sono estavam presentes em todos os pacientes no pré-operatório. Após a cirurgia, apenas um caso (Casc RD) ainda referia discreta sonolência diurna e nenhum descrevia pausas respiratórias ao dormir. A perda de peso, verificada por ocasião da polissonografia pós-operatória variou de 27 a $52 \mathrm{~kg}$, ou seja, $16.3 \%$ a $41.4 \%$ do peso inicial. Nos registros pré-operatórios os IA estavam elevados em todos os casos; o estágio 3 de sono encontrava-se reduzido em três pacientes e ausente em um; o estágio 4 estava ausente em todos os casos; o estágio REM mostrava-se reduzldo em três casos; queda de Sa02 abaixo dos parâmetros normais, durante as apnéias, ocorria em todos os pacientes (valor normal $>80 \%$ ). Todos apresentavam bradicardia acentuada durante os episódios apneicos, alternando com taquicardia após os episódios. No pós-operatório evidenciamos melhor dos IA em todos os pacientes, colocando três deles em níveis normais. Houve aumento do sono de ondas lentas e ao estágio REM; normalizaçāo da Sa02 em três casos; redução das arritmias cardíacas proporcional ao decréscimo das apnéias. $O$ caso $R D$ foi o único que persistiu com IA em niveis elevados, manteve quedas da Sa02 abaixo dos níveis normais e queixa de sonolência diurna.

\section{COMENTARIOS}

ASO é encontrada tanto em indivíduos obesos como não obesos mas é certamente mais comum nos primeiros 10. Postula-se que o tecido adiposo em vias aéreas superiores contribua para a reduçāo do lúmen a nivel do orofaringe 19 predispondo a oclusão apneica. Soma-se a outros fatores como: malformaçôes que alteram o calibre das vias aéreas superiores $13,15,25$; alteraçōes anatômicas adquiridas do orofaringe que interferem com a passagem aérea 2,6,15; hipotonia da musculatura faríngea e de genioglosso durante o sono, principalmente no estágio REM 7,20; decubito dorsal horizontal facilitando o deslocamento posterior da lingua 7. Por ser a obesidade apenas um dos componentes deste complexo multifatorial, nāo causa surpresa o fato de só raramente poder se eliminar a ASO apenas com redução de peso 3,16 , embora essa seja medida auxiliar útil nos casos submetidos a traqueostomia e uvulopalatofaringoplastia 22,23,24. A obesidade extrema também propicia disfunção respiratória caracterizada por hipoventilação alveolar $5,18,21$, que se soma as apnéias, agravando o quadro cardiopulmonar. 
Em nossa casuística, a avaliação clínica pré-operatória era típica da ASO com sonolência diurna, roncar intenso e pausas respiratórias durante o sono relatadas pela cônjuge 1,4,10,11. A melhora no pós-operatório a médio prazo, na amostra aqui relatada foi constatada pela redução do lA e consequiente aumento do sono ininterrupto, permitindo a ocorrência de estágios 3, 4 e REM. Com o decréscimo das apnéias houve melhora acentuada da $\mathrm{SaO}_{2}$ e das arritmias cardiacas.

A redução de peso mediante dietas apresenta a desvantagem de ser tratamento lento, principalmente ao se considerarem os casos com hipoventilação alveolar pelo peso excessivo superposta as ASO. E também uma forma terapêutica dificil de ser mantida, ou mesmo iniciada, e amiúde tais pacientes referem ter tentado inúmeras dietas, com parcos resultados. Gastroplastia é técnica cirúrgica estabelecida e relativamente simples, utilizada na reduçăo de peso $8,9,14,22$. Suas complicaçōes imediatas são incomuns, e compõem-se de perfuração gástrica, deiscência devido a obesidade extrema, náuseas e vômitos. $\mathrm{O}$ principal impecilho a longo prazo é a redilatação gástrica. $\mathrm{Na}$ pequena amostra aqui descrita, nāo observamos complicações, embora o seguimento seja ainda restrito. Nossos achados indicam que gastroplastia pode ser um tratamento alternativo valioso para a redução de peso em pacientes com ASO. A seleção desses casos é fundamental e, no momento, a indicação está reservada àqueles com ASO severa, obesidade intensa e cujas tentativas de restrição dietética tinham sido infrutiferas.

\section{RESUMO}

Gastroplastia foi realizada como forma terapêutica auxiliar para redução de peso em 4 pacientes com obesidade severa e apnéia obstrutiva durante o sono (ASO). As idades variavam de 31 a 54 anos e todos eram do sexo masculino. Em três casos a gastroplastia acompanhou-se de traqueostomia. Após a gastroplastia todos tiveram melhora da sonolência diurna e redução de peso em $16,7 \%$ a $40,9 \%$. Avaliaçōes polissonográficas de noite inteira realizadas no pré e pós-operatório (3-4 meses). Os registros pós-operatórios foram feitos com traqueostomia fechada e revelaram redução da freqüência das apnéias e aumento dos estágios 3, 4 e REM. Normalização dos índices de saturação arterial de oxigênio $\left(\mathrm{SaO}_{2}\right)$ foi constatada em três dos 4 casos. Esses dados sugerem que a gastroplastia pode ser utilizada como forma alternativa para redução de peso em casos selecionados de ASO complementando outros procedimentos cirúrgicos como a traqueostomia.

\section{SUMMARY}

\section{Gastroplasty as an adjunctive treatment for sleep apnea.}

Four male obese patients with obstructive sleep apnea were evaluated by polysomnography, both prior and 3-4 months following gastroplasty. The surgery was performed as an alternative weight loss treatment. These patients were selected for gastroplasty because they had severe obesity, obstructive 
sleep apnea with cardiopulmonary impairment and noncompliance on a weight loss diet. Tracheostomy was performed concomitantly in three cases. Preoperative recording demonstrated 21.2 to 100.3 apneas per hour of sleep; stage 3 was decreased in three and absent in one case; stage 4 was absent in every patient; stage REM was decreased in three cases; arterial oxygen saturation $\left(\mathrm{SaO}_{2}\right)$ was below $80 \%$ in every case during apenas. Follow-up recordings with occluded tracheostomy were obtained 3-4 months after surgery. The weight reduction varied from 16.3 to $41.4 \%$ of the initial weight. The recording documented normal sleep apnea indices in three cases and partial recovery in the remainder; increase in stages 3,4 and $\mathrm{REM}$; normal $\mathrm{SaO}_{2}$ in three out of 4 cases. These findings suggest that gastroplasty may be used as an alternative treatment for weight reduction in selected OSA patients.

\section{REFERENCIAS}

1. ASSOCIATION OF SLEEP DISORDERS CENTERS - Diagnostic elassification of sleep and arousal disorders. Sleep 2:1, 1979.

2. BAKER, S.R. \& ROSS, J. - Sleep apnea syndrome and supraglottic edema. Arch. Otolaryngol. $106: 486,1980$.

3. Charuzi, I.; OVNaT, A.: PEISER, J.; SATZ, H.; WEITZMaN, S. \& LAVIE, P. - The effect of surgical welght reduction on sleep quality in obesity-related sleep apnea syndrome. Surgery $97: 535,1985$.

4. ChAUdhARY, B.A. \& SPEIR, W.A. - Sleep apnea syndromes. South. med. J. $75: 39,1982$.

5. CHODOFF, P.; IMBEMBO, A.L. \& KNOWLES, C.L. - Massive weight loss folowing jejunoileal bypass: effects on pulmonary function. Surgery 81:399, 1977.

6. COCCAGNA, G.; DIDONATO, G. \& VERUCCHI, P. - Hypersomnia with periodic apneas in acquired micrognathia (a bird-like face syndrome). Arch. Neurol. 33:769, 1976.

7. DERMAN, S. \& KARACAN, I. - Sleep-induced respiratory disorders. Psyychiat. Ann. 9:411, 1979 .

8. FREEMAN, J.B. \& BURCHETT, H. — Failure rate with gastric partitioning for morbid obesity. Am. J. Surg. 145:113, 1983.

9. GRIFFEN, W.O.: YOUNG, L.V. \& STEVENSON, C.C. - A prospective comparison of gastric and jejuno-ileal bypass procedures for morbid obesity. Ann. Surg. 186:500, 1977.

19. GUILleminaUlT, C. - Sleep and breathing. In C. Guilleminault (ed.): Sleeping and waking disorders: indications and techniques. Addison-Wesley Publishing Co., Menlo Park, 1983, pg. 155.

11. GUilleminault, C.; CUMmiskey, J. \& DEMENT, W.C. - Sleep apnea syndrome: recent advances. Adv, in. Med. 26:347, 1980.

12. GUILleminAULT, C. \& DEMENT, W.C. - Sleep apriea syndromes and related sleep disorders. In C. Guilleminault (ed.): Sleep Apnea Syndromes. Allen B. Liss Publishing Co., New York, 1978, pg. 9.

13. IMES, C.K.; ORR, W.C.; SMITH, R.O. \& ROGERS, R.M. - Retrognathia and sleep apnea. J. amer. med. Assoc. 237:1596, 1977.

14. LINNER, J.H. - Comparative effectiveness of gastric bypass and gastroplasty. Arch. Surg. 117:695, 1982.

15. OLSEN, K.D.; SUH, K.W. \& STAATS, B.A. - Surgically correctable causes of sleep apnea syndrome. Otolaryngol. Head Neck Surg. 89:726, 1981.

16. PEISER, J.; LAVIE, P.; OVNAT, A. \& CHARUZZI, I. - Sleep apnea syndrome in the morbidly obese as an indication for weight reduction surgery. Ann. Surg. $199: 112,1984$. 
17. RECHTSCHAFFEN, A. \& KALES, A. - A Manual of Standardized Terminology, Techniques and Scoring System for Sleep Stages of Human Subjects. Brain Information Service/Brain Research Institute. University of California, Los Angeles, 1968.

18. REISIN, E. \& FROLICH, E. - Obesity: cardiovascular and respiratory pathophysiological alterations. Arch. int. Med. $141: 431,1981$.

19. ROCHESTER, D.F, \& ENSON, Y. - Current concepts in the obesity-hypoventilation syndrome: mechanical and circulatory factors. Amer. J. Med. 57:402, 1974.

20. ROJEWSKI, T.E.; SCHULLER, D.E.; SCHMIDT, H.S.; CLARK, R.W. \& POTTS, R.E. - Synchronous video recording of the pharyngeal airway and polysommograph in patients with obstrictive sleep apnea. Laryngoscope 92:246, 1982.

21. SANTESSON, J. \& NORDENTROM, $I$. - Pulmonary function in extreme obesity: influence of weight loss following intestinal shunt operation. Acta chir. scand. $482: 36,1978$.

22. SUGERMAN, H.J.; FAIRMAN, R.P. \& LINDEN, A.K. - Gastroplasty for respiratory insufficiency of obesity. Ann. Surg. 193:677, 1981.

23. SUGERMAN, H.J. \& FAIRMAN, R.P. - Massive weight loss will improve arterial oxygenation in selected patients. Anesthesiology 55:604, 1981.

24. VICTOR, D.W.; SARMIENTO, C.F.; YANTA, M. \& HALVERSON, J.D. - Obstructive sleep apnea in the murbidly obese: an indication for gastric bypass. Arch. Surg. 119:970, 1984.

25. WEITZMAN, E.D.; POLLACK, C.P. \& BOROWIECKI, B. - Hypersomnia-sleep apnea due to micrognathia. Arch. Neurol. 35:392, 1978.

Sleep Disorders Center, Ncurodiagnostics Department, Baptist Memorial Hospital S99 Madison Avenue - Memphis 38146 - U.S.A. 